\title{
ON NEWTON'S POLYGONS, GRÖBNER BASES AND SERIES EXPANSIONS OF PERTURBED POLYNOMIAL PROGRAMS
}

\author{
KONSTANTIN AVRACHENKOV \\ INRIA Sophia Antipolis, 2004 route des Lucioles, B.P. 93, 06902, France \\ VLADIMIR EJOV and JERZY A. FILAR \\ School of Mathematics and Statistics, University of South Australia \\ Mawson Lakes, SA 5095, Australia \\ E-mail: jerzy.filar@unisa.edu.au
}

\begin{abstract}
In this note we consider a perturbed mathematical programming problem where both the objective and the constraint functions are polynomial in all underlying decision variables and in the perturbation parameter $\varepsilon$.

Recently, the theory of Gröbner bases was used to show that solutions of the system of first order optimality conditions can be represented as Puiseux series in $\varepsilon$ in a neighbourhood of $\varepsilon=0$. In this paper we show that the determination of the branching order and the order of the pole (if any) of these Puiseux series can be achieved by invoking a classical technique known as the "Newton's polygon" and using it in conjunction with the Gröbner bases techniques.
\end{abstract}

1. Introduction. There are many real-world problems that are formulated as either linear or nonlinear mathematical programs. In a vast majority of cases it is assumed that the objective function and the constraints are fully and precisely known. However, that is rarely the case in applications. Hence a fundamental question that arises concerns the stability (or instability) of a solution when the problem is slightly perturbed.

A recent paper [5] considered solutions of the optimality conditions for the, equality constrained, mathematical program:

$$
\begin{array}{ll} 
& \min f(\mathbf{x}, \varepsilon) \\
\text { s.t. } & h_{i}(\mathbf{x}, \varepsilon)=0 ; i=1, \ldots, p .
\end{array}
$$

where $\mathbf{x} \in \Re^{n}, \varepsilon \in[0, \infty), f$, and $h_{i}$ 's are polynomial functions on $\Re^{n} \times[0, \infty)$. The case

2000 Mathematics Subject Classification: 34D15, 13P10, 65K10.

This research was supported by a grant from the Australian Research Council no. DP0343028.

The paper is in final form and no version of it will be published elsewhere. 
$\varepsilon=0$ corresponds to the underlying unperturbed program $(\mathrm{MP}(0))$. The parameter, $\varepsilon$, will be called the perturbation.

The problem of characterising solutions, $\mathbf{x}^{*}(\varepsilon)$, of $(\mathrm{MP}(\varepsilon))$ as functions of the perturbation parameter, $\varepsilon$ is closely related to the well established topics of sensitivity or post-optimality, or parametric analyses of mathematical programs. There is already a substantial volume of literature devoted to these topics (see for instance Bonnans and Shapiro [3], Fiacco [8], Gaitsgory and Pervozvanskii [18], Gal [10], Gal and Greenberg [11] and Levitin [16]). The approach proposed in Ejov and Filar [5] has been inspired by Jeroslow [12], [13], Eaves and Rothblum [7], Szczechla et al. [20] and Coulomb et al. [4].

The latter approach - based on the theory of complex analytic varieties (e.g. see Whitney [22]), Gröbner bases(e.g., see [1]) and series expansions - treats singular and regular perturbations in a unified way. In [5] Gröbner bases were used to construct Puiseux series solutions of the now classical Karush-Kuhn-Tucker conditions (the existence of such solutions was already established in [4]). Hence, it is now known that there exists a neighbourhood $\left(0, \varepsilon^{*}\right)$, an integer $m>0$ and a solution $\mathbf{x}^{*}(\varepsilon)$ of $(\mathrm{MP}(\varepsilon))$ in that neighbourhood that is expressible as a Laurent-Puiseux series of the form

$$
\mathbf{x}^{*}(\varepsilon)=\sum_{k=K}^{\infty} \varepsilon^{\frac{k}{m}} \mathbf{c}_{k} .
$$

In [5] the above was achieved by showing that Buchberger's algorithm in Gröbner bases theory can be used to characterise the variety of interest as the zero set of a system of bivariate polynomials. A classical result - due to Pusiseux (e.g., see [19], [6]) — concerns a Puiseux series solution of an irreducible bivariate polynomial. Hence, a somewhat tedious, reduction of a bivariate polynomial to its irreducible factors was proposed in [5].

This note is devoted to the problem of determination of the branching order and the order of the pole (if any) of the above Puiseux series with the help of Newton's polygons.

2. Polynomial programming setting. Consider a perturbed mathematical program

$$
\min f(\varepsilon, \mathbf{x})
$$

subject to

$$
h_{i}(\varepsilon, \mathbf{x})=0 ; i=1,2, \ldots, p
$$

where all functions are polynomials in the original decision variables $\mathbf{x}=x_{1}, x_{2}, \ldots, x_{n}$ and the single perturbation parameter $\varepsilon$. We are interested in the asymptotic behaviour of the solutions $x_{k}(\varepsilon)^{\prime}$ s as $\varepsilon \downarrow \varepsilon_{0}$, where $\varepsilon_{0}$ is naturally 0 , but also may be some other distinguished accumulation point of the perturbation parameter.

DEFinition 2.1. We shall say that a feasible point $(\varepsilon, x)$ is regular if the gradient vectors of the constraints are independent.

It is well known that at a regular feasible point $(\varepsilon, x)$ the first order optimality conditions are satisfied. That is, there exist Lagrange multipliers (dependent on $\varepsilon$ ) $\lambda_{1}, \lambda_{2}, \ldots, \lambda_{p}$ $\in \mathbb{R}$, not all zero, such that

$$
\sum_{i=1}^{p} \lambda_{i} \nabla h_{i}(\varepsilon, x)+\nabla f(\varepsilon, x)=0 .
$$


Hence, the system we are interested in consists of polynomial equations

$$
h_{i}(\varepsilon, \mathbf{x})=0, \quad i=1,2, \ldots, p ; \quad \sum_{i=1}^{p} \lambda_{i} \nabla h_{i}(\varepsilon, x)+\nabla f(\varepsilon, x)=0 .
$$

In particular, we are interested in solutions of $(2)$ that express $x_{j}(\varepsilon)$ as Puiseux series in $\varepsilon$ for $j=1, \ldots, n$.

To see that the latter arise naturally, consider a simple unconstrained problem of minimizing the function

$$
f\left(\varepsilon, x_{1}, x_{2}\right)=\frac{x_{1}^{4}}{4}+\frac{x_{2}^{4}}{4}+\frac{\varepsilon}{3} x_{1}^{3} x_{2}+\varepsilon x_{1} .
$$

We observe that the vanishing gradient conditions $\frac{\partial f}{\partial x_{1}}=\frac{\partial f}{\partial x_{2}}=0$ require the solution of simultaneous polynomial equations

$$
\begin{array}{r}
f_{1}=x_{1}^{3}+\varepsilon x_{1}^{2} x_{2}+\varepsilon=0, \\
f_{2}=x_{2}^{3}+\frac{\varepsilon}{3} x_{1}^{3}=0 .
\end{array}
$$

The generators polynomials $\left\{f_{1}, f_{2}\right\}$ do not compose a Gröbner basis ${ }^{1}$. To obtain a Gröbner basis for $F=\left\{f_{1}, f_{2}\right\}$ we first choose the pure lexicographic term order $x_{1} \prec$ $x_{2}$ (for different types of term orderings we refer a reader to [1] p. 19). The leading terms of $f_{1}$ and $f_{2}$ are $\varepsilon x_{1}^{2} x_{2}$ and $x_{2}^{3}$, respectively, with $\varepsilon x_{1}^{2} x_{2}$ being their least common multiple. Hence, the $S$-polynomial that is an algebraic combination of $f_{1}$ and $f_{2}$ with monomial coefficients in which the leading term cancels out (for the precise definition of $S$-polynomials see [1] p. 40) equals to

$$
s_{1}:=S\left(f_{1}, f_{2}\right)=x_{2}^{2} f_{1}-\varepsilon x_{1}^{2} f_{2}=x_{2}^{2} x_{1}^{3}+\varepsilon x_{2}^{2}-\frac{1}{3} \varepsilon^{2} x_{1}^{5} ;
$$

the next $S$-polynomial is

$$
s_{2}:=S\left(s_{1}, f_{1}\right)=-\frac{1}{3} \varepsilon^{3} x_{1}^{7}-2 \varepsilon x_{2} x_{1}^{3}-\varepsilon^{2} x_{2}-x_{2} x_{1}^{6} ;
$$

and, finally, $S$-polynomial

$$
s=S\left(s_{2}, f_{1}\right)=\left(1-\frac{1}{3} \varepsilon^{4}\right) x_{1}^{9}+3 \varepsilon x_{1}^{6}+3 \varepsilon^{2} x_{1}^{3}+\varepsilon^{3}
$$

is a bivariate polynomial in $\left(\varepsilon, x_{1}\right)$, that is an element of the Groebner basis of $F$ with respect to the term order $x_{1} \prec x_{2}$, which in Maple is denoted as gbasis $\left(F, p l e x\left(x_{1}, x_{2}\right)\right.$ ). Analogous construction for the term order $x_{2} \prec x_{1}$ leads to a bivariate polynomial in $\left(\varepsilon, x_{2}\right)$ that is,

$$
r=\left(\varepsilon^{4}-3\right) x_{2}^{9}+3 \varepsilon^{2} x_{2}^{6}-\varepsilon^{4} x_{2}^{3}+\frac{1}{9} \varepsilon^{6} .
$$

Had $s$ and $r$ been irreducible polynomials, they would have each determined a unique solution in a form of complex algebraic functions $x_{1}(\varepsilon)$ and $x_{2}(\varepsilon)$ that would admit a Puiseux expansion at a branching point in $\varepsilon$.

\footnotetext{
${ }^{1}$ Loosely speaking a Gröbner basis derived from a given set of polynomials is another set of polynomials that generate the same ideal as the original set but are easier to solve (see [1]).
} 
It turns out that in our case $s$ and $r$ are reducible. Nevertheless, the substitution $\varepsilon:=3 t^{3}$ leads to the factorisation

$$
s=-\left(3 x_{1}^{3} t^{4}-x_{1}^{3}-3 t^{3}\right)\left(9 x_{1}^{6} t^{8}+3 x_{1}^{6} t^{4}+x_{1}^{6}+9 t^{7} x_{1}^{3}+6 x_{1}^{3} t^{3}+9 t^{6}\right) .
$$

The first factor $f_{3}:=3 x_{1}^{3} t^{4}-x_{1}^{3}-3 t^{3}$ is irreducible and has the solution

$$
x_{1}=-\frac{\sqrt[3]{3} t}{\sqrt[3]{1-3 t^{4}}}, t=\sqrt[3]{\frac{\varepsilon}{3}}
$$

that admits a representation as a Puiseux series in $\varepsilon$ :

$$
x_{1}=-\sqrt[3]{3} t-\sqrt[3]{3} t^{5}+O\left(t^{9}\right)=-\varepsilon^{1 / 3}-3^{-4 / 3} \varepsilon^{5 / 3}+O\left(\varepsilon^{3}\right) .
$$

Let's suppose that the coordinates $\left(x_{1}, \varepsilon\right)$ of the initial point $x^{0}=\left(x_{1}, x_{2}, \varepsilon\right)$ satisfy the above solution for $x_{1}$. So, we can add $f_{3}$ to our original system $F$ in order to find the factor of $r$ that is compatible with $f_{3}$. To achieve this we change the term order to $x_{1} \prec x_{2}$ and consider the Gröbner basis gbasis $\left(F, p l e x\left(x_{2}, x_{1}\right)\right)$. This Gröbner basis contains a bivariate in $\left(x_{2}, \varepsilon\right)$ element $x_{2}^{3}\left(1-3 t^{4}\right)-3 t^{6}$, which is, in fact, a factor of $r{ }^{2}$ The solution

$$
x_{2}=\frac{\sqrt[3]{3} t^{2}}{\sqrt[3]{1-3 t^{4}}}, t=\sqrt[3]{\frac{\varepsilon}{3}},
$$

which equals $t x_{1}$, combined with (3) produces a solution of the original system $f_{1}=f_{2}=0$ through the initial point $x^{0}$, that can be expressed as a Puiseux series in $\varepsilon$ around $\varepsilon=0$ as

$$
x_{2}=\sqrt[3]{3} t^{2}+\sqrt[3]{3} t^{6}+O\left(t^{10}\right)=3^{-1 / 3} \varepsilon^{2 / 3}+3^{-5 / 3} \varepsilon^{2}+O\left(\varepsilon^{3}\right) .
$$

REMARK 2.1. In the above example it was a simple matter to obtain the needed irreducible factor $f_{3}$. However, in general, this not a trivial task. It is this observation that motivated the present note.

3. Reduction to bivariate polynomials. The polynomial system (2) defines a variety $W_{1}$, that is, a null-set of some ideal $I_{W_{1}}$ generated by polynomials $g_{i}(z, \eta), i=1,2, \ldots, \nu$. Adopt the term order $T_{1}:=z_{1} \prec z_{2} \prec \ldots z_{n}$ keeping $\eta$ as a parameter. Find the reduced Gröbner basis (see [1] p. 48) of $I_{W_{1}}$. To keep the notation simple we denote this basis by $G B^{(1)}\left(W_{1}\right)=\left\{g_{1}(z, \eta), \ldots, g_{t}(z, \eta)\right\}$. In the complex domain $z_{j}$ replaces the real variable $x_{j}$ and $\eta$ replaces $\varepsilon$.

The following key lemmata are proved in [5]. They demonstrate that the original system can be replaced by a decoupled system of bivariate polynomials in $\eta$ and in one of the $z_{j}$ variables.

LemMA 3.1. (i) One can order $g_{1}, \ldots, g_{t}$ so that $g_{1}$ is a univariate polynomial in the variable $z_{1}$, polynomial $g_{2}$ contains only the variables $z_{1}, z_{2}$, polynomial $g_{3}$ contains only $z_{1}, z_{2}, z_{3}$ and so forth until the polynomial $g_{n}$, containing $z_{1}, \ldots, z_{n}$. In particular, $t=n$.

(ii) The coefficients of $g_{i}, i=1, \ldots, t$ are rational functions in the $\eta$ variable.

\footnotetext{
${ }^{2}$ It could happen that the latter gbasis $\left(F, p l e x\left(x_{2}, x_{1}\right)\right)$ might consist of only the unit $\{1\}$, that would simply mean that $f_{3}$ does not have a compatible irreducible bivariate polynomial in $\left(x_{2}, \varepsilon\right)$ and we should have considered other irreducible factors of $s$.
} 
Consider polynomial $g_{1}(z, \eta)$. Having multiplied by the the least common multiple of the denominators of its coefficients we obtain a bivariate polynomial in $(z, \eta)$ that we denote by $\tilde{g}_{1}(z, \eta)$. This polynomial can be factorised into prime (irreducible) factors $([15])$ :

$$
\tilde{g}_{1}\left(z_{1}, \eta\right)=\prod_{1}^{\ell_{1}} p_{j}\left(z_{1}, \eta\right)
$$

Without loss of generality we assume that the initial point $\left(x_{0}, \varepsilon_{0}\right)$ belongs to the zero-set of $p_{1}\left(z_{1}, \eta\right)$, the first factor ${ }^{3}$ in $(7)$. We now add $p_{1}\left(z_{1}, \eta\right)$ to the $G B^{(1)}\left(W_{1}\right)$, change the term order to $T_{2}:=z_{2} \prec z_{1} \prec \ldots \prec z_{n}$ and construct the reduced Gröbner basis $G B^{(2)}\left(W_{1}\right)$ initiated by the set of generators $G B^{(1)}\left(W_{1}\right)$ and the term order $T_{2}$. By Lemma 3.1 the first element of $G B^{(2)}$ will be a univariate polynomial $g\left(z_{2}\right)$ with rational coefficients in $\eta$. Again, multiplying by the least common multiple of the coefficients' denominators and taking the irreducible factor $p_{2}\left(z_{2}, \eta\right)$ such that $\left(x_{0}, \varepsilon_{0}\right)$ belongs to its zero set, we obtain the second irreducible bivariate (in $z_{2}$ and $\eta$ ) polynomial that we can add to $G B^{(2)}$ to continue with the process. The uniqueness of the solution of a system of bivariate irreducible polynomial equations (see Section 5) ensures that this solution belongs to the original variety $W_{1}$. The above argument proves the following lemma.

LEMMA 3.2. In a neighbourhood of $\left(x_{0}, \varepsilon\right) \in S$ the variety $W_{1}$ is defined as a union of zero-sets of $\tau$ systems of $n$ irreducible bivariate polynomials $p_{1}^{i}\left(z_{1}, \eta\right), p_{2}^{i}\left(z_{2}, \eta\right), \ldots$, $p_{n}^{i}\left(z_{n}, \eta\right), i=1, \ldots, \tau$.

Note that the superscript $i$ in the above refers to a selection of one irreducible component from each of the product expression of the type $(7)$ for $\tilde{g}_{k}\left(z_{k}, \eta\right) ; k=1,2, \ldots n$. Thus, $\tau$ could be a large number.

For instance, in the preceding example an irreducible (in $z_{j}$ variables) bivariate system consisted of

$$
p_{1}^{1}\left(z_{1}, \eta\right)=3 z_{1}^{3} t^{4}-z_{1}^{3}-3 t^{3}, \quad p_{2}^{1}\left(z_{2}, \eta\right)=z_{2}^{3}\left(1-3 t^{4}\right)-3 t^{6} \quad \text { for } \quad t=\sqrt[3]{\frac{\eta}{3}} .
$$

The practically interesting perturbed polynomial programs are those that admit real solutions for real values of the perturbation parameter $\varepsilon$. Here we formulate a criterion for the existence of a real solution in terms of the Taylor or Laurent-Puiseux series coefficients.

Consider the original optimization problem (1) and its reduction through the complexification and the Gröbner bases' elimination procedure to a finite sequence of algebraic problems of the form

$$
\left\{Q_{j}\left(z_{j}, \eta\right)=0 ; j=1, \ldots, n\right\},
$$

for some irreducible bivariate polynomials $Q_{j}\left(z_{j}, \eta\right)$ of the degrees $\operatorname{deg}_{z_{j}} Q_{j}=m_{j}$. In [5] the following theorem was proved.

\footnotetext{
${ }^{3}$ The initial point could also belong to the zero set of some other polynomial in the product, say, $p_{j}\left(z_{1}, \eta\right)$. In this case the described algorithm should be applied to each possibility $p_{j}\left(z_{1}, \eta\right)=0$.
} 
TheOREM 3.1. The program (1) has a real solution $\mathbf{x}(\varepsilon)$ on some interval $\varepsilon \in\left(0, \varepsilon_{0}\right)$, $\varepsilon_{0}>0$ if and only if for at least in one of the algebraic reductions (8) every solution $z_{j}$ can be written as a Taylor or Laurent-Puiseux series with real coefficients:

$$
z_{j}(\varepsilon)=\sum_{k=0}^{\infty} c_{k}^{(j)} \varepsilon^{k}, c_{k}^{(j)} \in \mathbb{R},
$$

or

$$
z_{j}(\varepsilon)=\sum_{k=-k_{0}}^{\infty} c_{k}^{(j)} \varepsilon^{\frac{k}{m_{j}^{\prime}}}, c_{k}^{(j)} \in \mathbb{R},
$$

for some $k_{0}>0, m_{j}^{\prime}<m_{j}$ and for the choice of the branch $\varepsilon^{\frac{1}{m_{j}^{\prime}}}>0$.

Once again, we see that there is a need for a more systematic method of deriving irreducible factors and that such a method would be facilitated by an efficient determination of the orders $m_{j}$.

4. Irreducible factors by Gröbner bases. Consider a bivariate polynomial

$$
Q(z, \eta)=\sum_{\alpha+\beta \leq m} c_{\alpha \beta} z^{\alpha} \eta^{\beta}
$$

which is a polynomial in $(z, \eta)$ of degree $m>1$ with complex coefficients $c_{\alpha \beta}$. Without loss of generality we assume that $Q(0,0) \neq 0$, that is, $c_{00} \neq 0$; (this can always be achieved by moving the origin away from the zero set of $Q$ ). Having fixed two positive integers integers $m_{1}$ and $m_{2}=m-m_{1}$, we would like to find out if it is possible to represent $Q(z, w)$ as a product

$$
Q(z, \eta)=Q_{1}(z, \eta) Q_{2}(z, \eta)
$$

for some polynomials $Q_{1}=\sum_{\alpha+\beta \leq m_{1}} a_{\alpha \beta} z^{\alpha} \eta^{\beta}$ and $Q_{2}=\sum_{\alpha+\beta \leq m_{2}} b_{\alpha \beta} z^{\alpha} \eta^{\beta}$ of degrees $m_{1}$ and $m_{2}$, respectively. Without loss of generality we assume that $a_{00}=1$. Equating coefficients in (9) at each power product $z^{\alpha} \eta^{\beta}$ we obtain the following system of, at most, quadratic equations in the coefficients $\left(a_{\alpha \beta}, b_{\alpha \beta}\right)$, that determines all possible factorizations of $Q$ into two factors of prescribed degrees $m_{1}$ and $m_{2}$ :

$$
\sum_{\substack{\gamma+\lambda=\alpha \\ \delta+\mu=\beta}} a_{\gamma \delta} b_{\lambda \mu}= \begin{cases}c_{\alpha \beta}, & c_{\alpha \beta} \neq 0, \\ 0, & \text { otherwise }\end{cases}
$$

that we denote by $\mathcal{F}\left(Q, m_{1}, m_{2}\right)$.

Any solution $\left\{a_{\alpha \beta}\right\}$ and $\left\{b_{\alpha \beta}\right\}$ of $\mathcal{F}\left(Q, m_{1}, v_{2}\right)$ provides a factorization of $Q$ into factors of degrees $m_{1}$ and $m_{2}$. Under the assumption $a_{00}=1$ system (10) has, at most, finitely many solutions. If the solution set of $(10)$ in $\mathcal{F}\left(Q, m_{1}, m_{2}\right)$ is empty then that $Q$ can not be factorized into polynomials of degrees $m_{1}$ and $m_{2}$. Consider the ideal $I_{m_{1}, m_{2}}$ of the polynomials in the variables $\left\{a_{\alpha \beta}\right\}$ and $\left\{b_{\alpha \beta}\right\}$ generated by $\mathcal{F}\left(Q, m_{1}, m_{2}\right)$. Theorem 2.2.7 p. $63[1]$ states that $\mathcal{F}\left(Q, m_{1}, m_{2}\right)$ has no solutions if and only if any Gröbner basis of $I_{m_{1}, m_{2}}$ consists of just a unit. Because $Q$ has at most finitely many factors of the prescribed degrees, the only alternative case is when the solution set of $\mathcal{F}\left(Q, m_{1}, m_{2}\right)$ is finite. Then the same Theorem 2.2.7 p. 63 [1] states that if we adopt a pure lexicographic term order then the first element in the corresponding Gröbner basis will be univariate, 
the second will be bivariate, and so forth, that enables us to find the solutions $a_{\alpha \beta}, b_{\alpha \beta}$ precisely. Running this algorithm for $m_{1}=1, \ldots,\left[\frac{m}{2}\right]$ we either verify that $Q$ is irreducible or come across the smallest value $m_{1}$ that provides a factorization. Polynomial $Q_{1}$ of the degree $m_{1}$ then has to be irreducible. Applying the same algorithm to $Q_{2}$ and so on, we eventually obtain all other irreducible factors of $Q$.

It can be seen from the above that even finding the smallest value $m_{1}$ that provides the needed factorization is not a simple task. Fortunately, in the section below it will be seen that a classical technique due to Newton can supply this quantity.

5. Orders of Puiseux's series based on Newton's diagram. The above method is essentially based on the derivation of the system of irreducible bivariate polynomials. However, as one can see from the example of Section 2, it might be the case that the Gröbner basis elimination algorithm produces a system of reducible bivariate polynomials. In the preceding section it was shown that one can again apply Gröbner bases to factorize these into irreducible components. However, this approach is computationally demanding. In the present section we propose an alternative approach based on the Newton diagram technique. Since the latter is a classical construction (e.g., see [21]) we merely outline it here and illustrate various steps on the simple example of Section 2.

Here we permit that some polynomials in our bivariate system are reducible. In particular, in our example the reducible bivariate system consists of:

$$
\begin{aligned}
& s=\left(1-\frac{1}{3} \eta^{4}\right) z_{1}^{9}+3 \eta z_{1}^{6}+3 \eta^{2} z_{1}^{3}+\eta^{3}=0, \\
& r=\left(\eta^{4}-3\right) z_{2}^{9}+3 \eta^{2} z_{2}^{6}-\eta^{4} z_{2}^{3}+\frac{1}{9} \eta^{6}=0 .
\end{aligned}
$$

Let us consider a generic bivariate polynomial equation of the above form

$$
a_{n}(\eta) z^{n}+a_{n-1}(\eta) z^{n-1}+\ldots+a_{1}(\eta) z+a_{0}(\eta)=0,
$$

where coefficents $a_{k}(\eta), k=0, \ldots, n$ are polynomials of the perturbation parameter $\eta$. Namely,

$$
a_{k}(\eta)=a_{k}^{(0)}+\eta a_{k}^{(1)}+\ldots+\eta^{p_{k}} a_{k}^{\left(p_{k}\right)},
$$

for $k=0, \ldots, n$ with at least one $a_{k}^{(0)} \neq 0$.

To obtain the Puiseux series in $\eta$ of a solution of (11), we substitute (12) and the following Ansatz power series

$$
z(\eta)=z^{(1)} \eta^{\alpha}+z^{(2)} \eta^{\beta}+\ldots,
$$

where $\alpha$ and $\beta$ are rational numbers and $\alpha<\beta$, into (11). We note that the terms with the lowest degree of $\eta$ can be only among

$$
a_{0}^{\left(\rho_{0}\right)} \eta^{\rho_{0}}, \quad \ldots \quad a_{k}^{\left(\rho_{k}\right)}\left(z^{(1)}\right)^{k} \eta^{\rho_{k}+k \alpha}, \quad \ldots \quad a_{n}^{\left(\rho_{n}\right)}\left(z^{(1)}\right)^{n} \eta^{\rho_{n}+n \alpha},
$$

with

$$
\rho_{k}=\min \left\{i \mid a_{k}^{(i)} \neq 0\right\},
$$

for $k \in\{0,1, \ldots, n\}$ and $a_{k}(\eta) \not \equiv 0$. 
For instance, in the case of the $s$ polynomial in our example we have that $\rho_{0}=3$, $\rho_{3}=2, \rho_{6}=1$ and $\rho_{9}=0$. Similarly, in the case of the $r$ polynomial in our example we have that $\rho_{0}=6, \rho_{3}=4, \rho_{6}=2$ and $\rho_{9}=0$.

In order for lower powers in $\eta$ in (11) to cancel (after the above substitutions are made to replace $z$ ), it is needed that at least two powers among

$$
\rho_{0}, \quad \ldots \quad, \rho_{k}+k \alpha, \quad \ldots \quad, \rho_{n}+n \alpha
$$

coincide and the remaining powers be larger. Of course, one can follow a brute force search procedure. However, the Newton diagram approach makes the search much easier.

To construct a Newton diagram, we plot the collection of points $\left(0, \rho_{0}\right), \ldots\left(k, \rho_{k}\right), \ldots$ $\left(n, \rho_{n}\right)$ on a plane for $k \in\{0,1, \ldots, n\}$ and $a_{k}(z) \not \equiv 0$. Next let us draw a line through the point $\left(0, \rho_{0}\right)$ which coincides with the ordinate axis and rotate this line counterclockwise around $\left(0, \rho_{0}\right)$ until it touches one of the other points, say $\left(l, \rho_{l}\right)$. In fact, several points may fall on this line. Then, we choose the point on the line with the largest abscissa and draw a line through it parallel to the ordinate axis and again rotate it counterclockwise until it touches another point in the collection. Continuing in this manner, we obtain a lower envelope of the convex hull of the points $\left(0, \rho_{0}\right), \ldots\left(k, \rho_{k}\right) \ldots\left(n, \rho_{n}\right)$. This is the Newton diagram (it is also often called Newton polygon). In the case of our $s$ polynomial given above the Newton diagram is simply Figure 1.

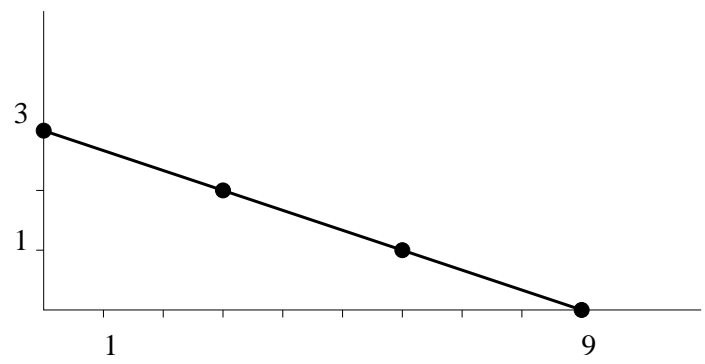

Fig. 1. Newton diagram for polynomial $s$

Now we note that the tangents of the angles between the segments of the Newton polygon and the negative direction of the abscissa give possible values for $\alpha$. Indeed, consider a segment and some point from the collection $\left(0, \rho_{0}\right), \ldots\left(k, \rho_{k}\right), \ldots\left(n, \rho_{n}\right)$ that does not lie on this segment. If we draw a line through that point parallel to the segment, the line will be above the segment, by construction. Hence, the powers of the terms corresponding to the points which do not belong to the segment are higher than the powers of the terms corresponding to the segment points.

Of course, in the case of our $s$ polynomial, we see from Figure 1 that there is only one segment with tangent of $\alpha=1 / 3$. Hence $m=3$ is the branching order of the corresponding Puiseux series solution.

The descending segments of the Newton polygon yield asymptotics for the roots that approach zero as the perturbation parameter goes to zero; and the ascending segments of the Newton polygon yield asymptotics for the roots that go to infinity as $\eta$ goes to 
zero. Note that in the case of the $s$ polynomial in our example, there are no ascending segments and hence the Puiseux series has no singularity at $\eta=0$.

Once $\alpha$ is determined, we can proceed to the calculation of the coefficient $z^{(1)}$. Consider a segment with the points $\left(k, \rho_{k}\right)$ such that $\rho_{k}+k \alpha=\sigma=$ const. In order for the lower power terms to cancel after the substitution of (13) into (11), $z^{(1)}$ should satisfy the polynomial equation

$$
\sum_{k: \rho_{k}+k \alpha=\sigma} a_{k}^{\left(\rho_{k}\right)}\left(z^{(1)}\right)^{k}=0 .
$$

The number of the non-zero roots in the above equation is equal to the length of the projection of the segment on the abscissa axis. Since the sum of all projections is equal to $n$, we obtain asymptotic expansions for all the roots of the polynomial (11).

Continuing with the application of the Newton diagram approach to the polynomial $s$ we observe from Figure 1 and the preceding calculations that

$$
z_{1}(\eta)=z_{1}^{(1)} \eta^{1 / 3}+\ldots
$$

The substitution of the above series into polynomial $s$ gives a polynomial equation for $z_{1}^{(1)}$.

$$
\begin{gathered}
\left(z_{1}^{(1)}\right)^{9}+3\left(z_{1}^{(1)}\right)^{6}+3\left(z_{1}^{(1)}\right)^{3}+1=0, \\
\left(\left(z_{1}^{(1)}\right)^{3}+1\right)^{3}=0 .
\end{gathered}
$$

The above equation indeed has nine complex roots (equal to the length of the projection of the relevant line segment on the horizontal axis). However, it has only one real solution, that is $z_{1}^{(1)}=-1$.

The procedure for determination of $\beta$ and $z^{(2)}$ is completely analogous. The only difference is that we would be constructing an upper (rather than the lower) envelope of the convex hull of the relevant points.

\section{References}

[1] W. Adams and P. Lostaunau, An Introduction to Gröbner Bases, Graduate Studies in Mathematics, vol. 3, AMS, 1994.

[2] J. M. Borwein and A. S. Lewis, Convex Analysis and Nonlinear Optimization, SpringerVerlag, New York, 2000.

[3] J. F. Bonnans and A. Shapiro, Perturbation Analysis of Optimization Problems, SpringerVerlag, New York, 2000.

[4] J.-M. Coulomb, J. Filar and W. Szchechla, Asymptotic analysis of perturbed mathematical programs, Journal of Mathematical Analysis and Applications 251 (2000), 132-156.

[5] V. Ejov and J. A. Filar, Gröbner bases in asymptotic analysis of perturbed polynomial programs, Math. Meth. Oper. Res., to appear.

[6] J. L. Coolidge, A Treatise on Algebraic Plane Curves, Dover, New York, 1959, p. 207.

[7] B. C. Eaves and V. G. Rothblum, A theory of extending algorithms for parametric problems, Math. of Oper. Res. 14 (1989), 502-533.

[8] A. V. Fiacco (ed.), Optimization with data perturbations, Ann. OR 27 (1990).

[9] O. Forster, Lectures on Riemann Surfaces, Springer-Verlag, New York, 1981. 
[10] T. Gal, Linear parametric programming - a brief survey, Math. Program. Study 21 (1984), 43-68.

[11] T. Gal and H. J. Greenberg (eds.), Advances in Sensitivity Analysis and Parametric Programming, Kluwer Academic Publishers, 1997.

[12] R. G. Jeroslow, Asymptotic linear programming, Oper. Res. 21 (1973), 1128-1141.

[13] R. G. Jeroslow, Linear programs dependent on a single parameter, Disc. Math. 6 (1973), 119-140.

[14] T. Kato, Perturbation Theory for Linear Operators, Springer-Verlag, Berlin, 1995.

[15] S. Lang, Algebra, Addison-Wesley, 1965.

[16] E. S. Levitin, Perturbations Theory in Mathematical Programming and its Applications, Wiley, New York, 1994.

[17] D. G. Luenberger, Linear and Nonlinear Programming, Addison-Wesley, 1984.

[18] A. A. Pervozvanskii and V. G. Gaitsgori, Theory of Suboptimal Decisions, Kluwer Academic Publishers, Dordrecht, 1988.

[19] V. Puiseux, Recherches sur les fonctions algébriques, J. Math. Pures Appl. 15 (1850), 207.

[20] W. W. Szczechla, S. A. Connell, J. A. Filar and O. J. Vrieze, On the Puiseux series expansion of the limit discount equation of stochastic games, SIAM J. Control Optim. 35 (1997), 860-875.

[21] M. M. Vainberg and V. A. Trenogin, Theory of Branching of Solutions of Non-linear Equations, Noordhoff International Publishing, Leyden, 1974.

[22] H. Whitney, Complex Analytic Varieties, Addison-Wesley, 1972. 